\title{
Author Correction: Cardioprotection induced in a mouse model of neuropathic pain via anterior nucleus of paraventricular thalamus
}

\author{
Yi-Fen Cheng ${ }^{1,2}$, Ya-Ting Chang ${ }^{2,3}$, Wei-Hsin Chen ${ }^{2}$, Hsi-Chien Shih², Yen-Hui Chen², Bai-Chuang Shyu ${ }^{2}$ \\ \& Chien-Chang Chen $1,2,4$
}

Correction to: Nature Communications https://doi.org/10.1038/s41467-017-00891-z, published online 10 October 2017

The original version of this Article contained an error in the affiliation of the second author, Ya-Ting Chang. The correct affiliations for Ya-Ting Chang are Institute of Biomedical Sciences, Academia Sinica, Taipei 115, Taiwan and International Graduate Program in Molecular Medicine, National Yang-Ming University and Academia Sinica, Taipei 115, Taiwan.

Published online: 23 April 2018

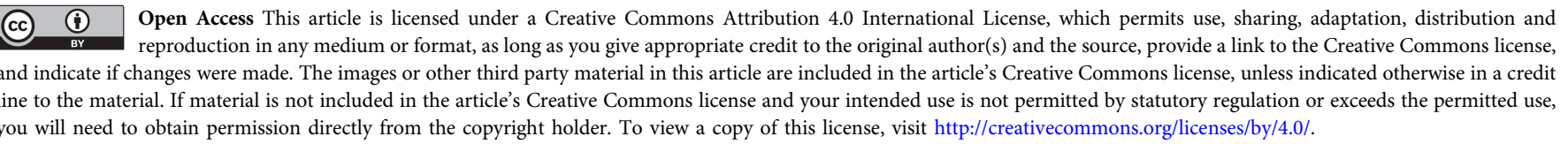
you will need to obtain permission directly from the copyright holder. To view a copy of this license, visit http://creativecommons.org/licenses/by/4.0/.

(c) The Author(s) 2018

\footnotetext{
${ }^{1}$ Graduate Institute of Life Sciences, National Defense Medical Center, Taipei 114, Taiwan. ${ }^{2}$ Institute of Biomedical Sciences, Academia Sinica, Taipei 115, Taiwan. ${ }^{3}$ International Graduate Program in Molecular Medicine, National Yang-Ming University and Academia Sinica, Taipei 115, Taiwan. ${ }^{4}$ Molecular Medicine Program, Taiwan International Graduate Program, Institute of Biomedical Sciences, Academia Sinica, Taipei 115, Taiwan. Correspondence and requests for materials should be addressed to C.-C.C. (email: ccchen@ibms.sinica.edu.tw)
} 